\title{
Aspects of two-dimensional dilaton gravity, dimensional reduction, and holography
}

\author{
K. Narayan \\ Chennai Mathematical Institute, SIPCOT IT Park, Siruseri 603103, India
}

(Received 18 November 2020; accepted 11 June 2021; published 16 July 2021)

\begin{abstract}
We discuss aspects of generic two-dimensional dilaton gravity theories. The two-dimensional geometry is in general conformal to the two-dimensional anti-de Sitter space $\left(\mathrm{AdS}_{2}\right)$ and has IR curvature singularities at zero temperature; this can be regulated by a black hole. The on-shell action is divergent; we discuss the holographic energy-momentum tensor by adding appropriate counterterms. For theories obtained by dimensional reduction of the gravitational sector of higher dimensional theories, for instance higher dimensional AdS gravity as a concrete example, the two-dimensional description dovetails with the higher dimensional one. We also discuss more general theories containing an extra scalar field which now drives nontrivial dynamics. Finally we discuss aspects of the two-dimensional cosmological singularities discussed in earlier work. These studies suggest that generic two-dimensional dilaton gravity theories are somewhat distinct from Jackiw-Teitelboim (JT) gravity and theories "near JT".
\end{abstract}

DOI: $10.1103 /$ PhysRevD.104.026007

\section{INTRODUCTION}

Dilaton gravity in two-dimensions has been under active investigation in recent years, in part following discussions of nearly the two-dimensional anti-de Sitter( $\left.\mathrm{AdS}_{2}\right)$ holography [1-5] (reviewed in [6-8]) and more recently those of Jackiw-Teitelboim (JT) gravity $[9,10]$ being dual to ensembles [11], with further development in [12-21]. It is well known that $\mathrm{AdS}_{2}$ arises in the near horizon geometry of extremal black holes and branes [6-8]. However twodimensional dilaton gravity per se arises quite generically from the two-dimensional subsector of higher dimensional gravity on spaces of the form $\mathcal{M}_{2} \times X^{d}$ upon Kaluza-Klein compactification over the compact space $X^{d}$.

It is interesting to ask how these generic dilaton gravity theories compare with JT gravity, i.e., if they are "near JT". Here we study certain aspects of generic two-dimensional dilaton-gravity theories of the form

$$
S=\frac{1}{16 \pi G_{2}} \int d^{2} x \sqrt{-g}(\phi \mathcal{R}-U(\phi))
$$

containing the two-dimensional dilaton coupling to gravity and a general dilaton potential. For JT gravity, the potential is $U=-2 \phi$. From the equations of motion,

Published by the American Physical Society under the terms of the Creative Commons Attribution 4.0 International license. Further distribution of this work must maintain attribution to the author(s) and the published article's title, journal citation, and DOI. Funded by SCOAP .

$$
g_{\mu \nu} \nabla^{2} \phi-\nabla_{\mu} \nabla_{\nu} \phi+\frac{g_{\mu \nu}}{2} U=0, \quad \mathcal{R}-\frac{\partial U}{\partial \phi}=0 .
$$

For $U \sim \phi^{n}$ asymptotically, the curvature diverges when the dilaton is vanishingly small,

$$
\mathcal{R} \sim \phi^{n-1} \stackrel{\phi \rightarrow 0}{\longrightarrow} \infty, \quad n<1 .
$$

The two-dimensional geometry is generically conformally $\mathrm{AdS}_{2}$ and the conformal factor leads to this (IR) singularity; this is in the zero temperature theory. Another consequence of the dilaton equation of motion is that the bulk on-shell action has a UV divergence where $\phi$ grows large,

$$
\begin{aligned}
S^{o . s .} & =\frac{1}{16 \pi G_{2}} \int d^{2} x \sqrt{-g}\left(\phi \partial_{\phi} U-U\right) \\
& \sim \frac{1}{16 \pi G_{2}} \int d^{2} x \sqrt{-g}(n-1) U .
\end{aligned}
$$

By comparison, in JT gravity the metric is $\mathrm{AdS}_{2}$ with constant curvature, and the potential is linear so the on-shell action vanishes.

In some sense, the theories (1) are reminiscent of certain nonrelativistic theories; e.g., hyperscaling violating (hvLif) theories conformal to AdS (or Lifshitz) exhibit curvature singularities in the zero temperature metric (see $[22,23]$ for reviews of various aspects of nonrelativistic holography). These are best regarded as effective gravity theories valid in certain intermediate regimes. For theories with gauge/string realizations, a UV complete description emerges in the latter. For instance some of these can be obtained from the 
supergravity description of nonconformal $D p$-branes [24] upon dimensional reduction on the transverse $S^{8-p}$ sphere [25]. While the effective hvLif theories here exhibit such singularities, the higher dimensional description is well behaved; the singularities arise from the compactification.

So far our discussion of (1) is bottom-up. Looking topdown, similar arguments apply at least when theories (1) arise by dimensional reduction from well-behaved theories in higher dimensions. A prototypical example in this regard is the reduction of higher dimensional gravity with a cosmological constant. Restricting to just this gravity sector alone, and focusing on a negative cosmological constant gives the action

$$
\begin{gathered}
S_{D}=\frac{1}{16 \pi G_{D}} \int d^{D} x \sqrt{-g^{(D)}}\left(\mathcal{R}^{(D)}-2 \Lambda\right), \\
\Lambda=-\frac{1}{2} d_{i}\left(d_{i}+1\right), \quad D=d_{i}+2 .
\end{gathered}
$$

We use reduction conventions of [26-28] (see also [29-32] for reviews). Assuming translational and rotational invariance in the $d_{i}$ boundary spatial directions (for theories with holographic duals) leads to the form $\mathcal{M}_{2} \times X^{d_{i}}$ for the higher dimensional space. For simplicity, we take $X^{d_{i}}$ to be a torus $T^{d_{i}}$ and compactify with the reduction ansatz

$$
d s_{D}^{2}=g_{\mu \nu}^{(2)} d x^{\mu} d x^{\nu}+\phi^{\frac{2}{d_{i}}} d \sigma_{d_{i}}^{2} ; \quad g_{\mu \nu}=\phi^{\frac{d_{i}-1}{d_{i}}} g_{\mu \nu}^{(2)} \text {. }
$$

The reduction and the Weyl transform $g^{(2)} \rightarrow g$ above gives the two-dimensional dilaton gravity theory

$S=\frac{1}{16 \pi G_{2}} \int d^{2} x \sqrt{-g}(\phi \mathcal{R}-U(\phi)), \quad U=2 \Lambda \phi^{1 / d_{i}}$,

See Appendix A for some details. The two-dimensional space here contains time and the radial coordinate, while the $d_{i}=D-2$ spatial coordinates define the "transverse" part of the bulk spacetime; in theories with holographic duals, $d_{i}$ is the spatial dimension of the boundary field theory. Note that the $\mathrm{AdS}_{3}$ also leads to $U=-2 \phi$. The dilaton defined as in (6) is the transverse area in the higher dimensional space $g_{i i}^{(D-2) / 2}=\phi$. The higher dimensional background here is $\operatorname{AdS}_{D}$ (Poincaré) with $d s^{2}=\frac{1}{r^{2}}\left(-d t^{2}+d r^{2}\right)+\frac{1}{r^{2}} d x_{i}^{2}$, of the general form (6).

The theory (7) is of the general form (1); the twodimensional theory is conformally $\mathrm{AdS}_{2}$ and the zero temperature theory has a curvature singularity (Sec. II) and the on-shell action is of the form (4) with $n=\frac{1}{d_{i}}$. Of course, operationally both of these are curable in (7) and more generally in (1); the curvature singularity is regulated by introducing a black hole horizon (Sec. II A) and the on-shell action is regulated by adding appropriate counterterms which render calculations of holographic observables reasonable (Sec. III and Sec. IVA).

These aspects are of course well known in the extensive studies of higher dimensional AdS/CFT [33-36] over the years; here we simply note that these two-dimensional theories exhibit similar features. In particular, some aspects of these sorts of two-dimensional dilaton gravity theories have also been studied e.g., [37]. Among other things, this describes the role in these theories of generalized conformal structure [38] (a symmetry under scaling transformations involving Weyl rescaling of the metric along with appropriate scalings of additional scalars representing running couplings), studied for nonconformal branes in [39-41] where various aspects of the holographic dictionary were described. Recalling our earlier comments on nonconformal branes and nonrelativistic theories, we see that the above features also apply in some of the present discussions stemming from parallels with the nontrivial dilaton profile which from (6) is part of the higher dimensional metric (with the associated higher dimensional symmetries).

Thus overall, although two-dimensional, (7) and more generally (1) is apparently encoding higher dimensional gravity intrinsically, this is quite different from the near extremal near horizon $\mathrm{AdS}_{2} \times X$ throats in extremal objects, where the $X$-compactification in fact leads to an intrinsically two-dimensional theory with a clear separation of scales. To put this in perspective, we note that the gravity subsector, taken stand-alone, is universal to all string/M theories on $\operatorname{AdS}_{D} \times X^{10 / 11-D}$. This is usually understood to be UV complete only if the entire higher dimensional (string/M) upstairs theory is included. From this point of view, it should not be surprising that these two-dimensional theories exhibit the divergences above; they are perhaps best regarded as UV incomplete low energy effective theories universal to all UV completions $\mathrm{AdS}_{D} \times X$ upstairs, so they are thermodynamic, akin to an ensemble. These features appear generic to twodimensional dilaton gravity theories of the form (1), with general (nonlinear) potentials. They also arise with additional matter, e.g., extra scalars that drive dynamics (Sec. IV). These studies suggest that generic twodimensional dilaton gravity theories are somewhat distinct from JT gravity and theories "near JT" which appear special. In what follows we will describe this in greater detail, with a discussion in Sec. V.

\section{ASPECTS OF GENERIC TWO-DIMENSIONAL DILATON GRAVITY}

We now describe in more detail aspects of the twodimensional theory (1). Using conformal gauge and combining the various Einstein equation components, the equations of motion (2) become 


$$
\begin{aligned}
& d s^{2}=g_{\mu \nu} d x^{\mu} d x^{\nu}=e^{f(r)}\left(-d t^{2}+d r^{2}\right), \\
& -\partial_{r}^{2} \phi+\partial_{r} f \partial_{r} \phi=0, \quad \partial_{r}^{2} \phi+e^{f} U=0, \\
& -\partial_{r}^{2} f-e^{f} \partial_{\phi} U=0 .
\end{aligned}
$$

It is adequate to exclude time dependence here; later we will discuss nontrivial time dynamics as well in the presence of an extra scalar. Solutions to these can be expressed in terms of a general prepotential $\int d \phi^{\prime} U\left(\phi^{\prime}\right)$ [42] (see also [43], Appendix E). In cases where $U(\phi)$ is a $\phi$-monomial, solutions can be found by employing a power-law scaling ansatz for $\phi, e^{f}$, and then solving the algebraic equations that result from (8); this was found to be useful in [28]. For the theory (1), a monomial potential gives from (8)

$$
\begin{aligned}
& U=A \phi^{n} ; \quad \phi=r^{m}, \quad e^{f}=r^{b} \Rightarrow \\
& (-m(m-1)+b m) r^{m-2}=0, \quad m(m-1) r^{m-2}+A r^{b+m n}=0, \quad b r^{-2}-A n r^{b+m(n-1)}=0, \\
& \Rightarrow \quad m(b-m+1)=0, \quad b+m(n-1)+2=0, \quad m(m-1)=-A, \quad b=A n .
\end{aligned}
$$

We require the $r$-exponents to match for a nontrivial solution valid for all $r$; this gives e.g., $m-2=b+m n$, and the remaining equations then follow. (The length scale we have suppressed can be reinstated when required) With $m \neq 0$, we obtain $m=b+1$ and thereby

$m=-\frac{1}{n}, \quad b=-\frac{1}{n}-1, \quad$ and $\quad A=-\frac{1}{n}\left(\frac{1}{n}+1\right)$,

as the unique solution, which can be checked to satisfy all the equations in (9). The last equation here is a condition that the parameters $A, n$, in the potential $U$ must satisfy for consistency of this solution to the theory. Thus the twodimensional background solution to (1) is

$\phi=\frac{1}{r^{1 / n}}, \quad d s^{2}=\frac{1}{r^{1+\frac{1}{n}}}\left(-d t^{2}+d r^{2}\right)=\frac{1}{r^{\frac{1}{n}-1}} d s_{\mathrm{AdS}_{2}}^{2}$.

The curvature (2) is

$$
\mathcal{R}=A n \phi^{n-1}=-\left(1+\frac{1}{n}\right) r^{\frac{1}{n}-1},
$$

giving an IR singularity in the deep interior at $r \rightarrow \infty$ for $n<1$ and at $r \rightarrow 0$ if $n>1$. JT gravity is the case $n=1$, the space being $\mathrm{AdS}_{2}$, the metric being $d s^{2}=\frac{1}{r^{2}}\left(-d t^{2}+d r^{2}\right)$ with constant curvature $\mathcal{R}=-2$.

Thus for $n \neq 1$, the two-dimensional geometry is conformal to $\mathrm{AdS}_{2}$ : for $n<1$ the conformal factor becomes larger towards the boundary $r \rightarrow 0$, so the space there "flattens". As $\phi$ grows to large field values, the curvature $\mathcal{R} \rightarrow 0$. The conformally $\operatorname{AdS}_{2}$ geometry (11) is akin to a nonconformal $D p$-brane geometry [24]; the corresponding Euclidean space is

$$
d s_{E}^{2} \sim d \rho^{2}+\rho^{\frac{2(1+n)}{1-n}} d t_{E}^{2}, \quad \rho \sim r^{\frac{1-1 / n}{2}} ; \quad \mathcal{R} \sim-\frac{1}{\rho^{2}} .
$$

In general, a conformally $\mathrm{AdS}_{2}$ metric $d s^{2}=\frac{e^{F(r)}}{r^{2}}\left(d t_{E}^{2}+d r^{2}\right)$ has curvature $\mathcal{R}=-e^{-F}\left(2+r^{2} F^{\prime \prime}\right)$ with a singularity as $e^{F} \rightarrow 0($ as $r \rightarrow \infty)$ unless $F=2 \log r$ (flat space) or $F=0$ $\left(\mathrm{AdS}_{2}\right)$. More general dilaton potentials can be used to find classical solutions to the equations (2): in general they will lead to conformally $\mathrm{AdS}_{2}$ spaces with singularities.

\section{A. Regulating with a black hole}

There are parallels with the IR curvature singularity described above for $n<1$ and similar singularities in hyperscaling violating theories: on the left below is the zero temperature metric

$$
\begin{aligned}
& d s^{2}=r^{2 \theta / d_{i}}\left(\frac{-d t^{2}+d r^{2}+d x_{i}^{2}}{r^{2}}\right) \rightarrow \\
& d s^{2}=\frac{r^{2 \theta / d_{i}}}{r^{2}}\left(-f(r) d t^{2}+d x_{i}^{2}+\frac{d r^{2}}{f(r)}\right) .
\end{aligned}
$$

Here the exponent $\theta<0$ and the conformal factor leads to a curvature singularity $\mathcal{R} \sim r^{-2 \theta / d_{i}}$ as $r \rightarrow \infty$. More general nonrelativistic theories include a Lifshitz exponent as well in the form $-\frac{d t^{2}}{r^{2 z}}+\cdots$ above and $\theta$ can be positive as well. These also have singularities with either curvatures or tidal forces diverging (see $[22,23]$ which review various aspects of nonrelativistic holography). In general these are best regarded as effective theories valid in some intermediate regimes; beyond these some further completion is required. In fact nonconformal $D p$-branes upon reduction on the transverse $S^{8-p}$ sphere give (14) with $\theta=p-\frac{9-p}{5-p}$ and $d_{i}=p$ [25]; the higher dimensional description is of course well known in string theory, with rich phase diagrams [24]. Similar features arise in the string realizations in [44].

At the low energy effective level, the metric on the left above can be de-singularized by introducing a black hole/ brane: the metric on the right in (14) is at finite temperature with a blackening factor $f(r) \sim 1-\frac{r^{\#}}{r_{0}^{\#}}$ that vanishes at a 
horizon $f\left(r_{0}\right)=0$. Now the space is smooth at the Hawking periodicity, where the Euclidean geometry has no conical singularity.

The behavior of the conformally $\mathrm{AdS}_{2}$ space (13) is very similar; the zero temperature theory is singular at the IR horizon $r \rightarrow \infty$ due to the conformal factor. As above, we can regulate this via a black hole horizon,

$$
d s^{2}=e^{f(r)}\left(-d t^{2}+d r^{2}\right) \longrightarrow e^{f(r)}\left(-H(r) d t^{2}+\frac{d r^{2}}{H(r)}\right) ;
$$$$
\text { with } H \stackrel{r \rightarrow 0}{\longrightarrow} 1, \quad H \stackrel{r \rightarrow r_{0}}{\longrightarrow} 0 \text {. }
$$

The Einstein equations (2) now give ${ }^{1}$

$$
-\partial_{r}^{2} \phi+f^{\prime} \partial_{r} \phi=0, \quad \partial_{r}\left(H \partial_{r} \phi\right)+e^{f} U=0 .
$$

The first equation implies $\partial_{r} \phi \propto e^{f}$. Now since we are thinking of the blackening factor $H(r)$ as a finite temperature regulator to the original space, these equations must hold simultaneously along with (8) after imposing the $H$ boundary conditions in (15), this gives

$$
\begin{aligned}
\partial_{r}\left(H \partial_{r} \phi\right) & =-e^{f} U=\partial_{r}^{2} \phi \quad \rightarrow \\
\partial_{r}\left((H-1) \partial_{r} \phi\right) & =0 \quad \rightarrow \quad H=1-e^{-f+f_{0}} .
\end{aligned}
$$

Near the horizon $r \sim r_{0}$, the Euclidean space becomes $\left(H_{0}^{\prime}=f_{0}^{\prime}<0\right)$

$$
\begin{gathered}
d s^{2} \stackrel{r \rightarrow r_{0}}{\longrightarrow} e^{f_{0}}\left(\left.H^{\prime}\right|_{0}\left(r-r_{0}\right) d t_{E}^{2}+\frac{d r^{2}}{\left.H^{\prime}\right|_{0}\left(r-r_{0}\right)}\right) \\
\rightarrow \frac{f_{0}^{\prime 2}}{4} \rho^{2} d t_{E}^{2}+d \rho^{2} ; \quad \& \phi \rightarrow \phi_{h},
\end{gathered}
$$

which has no conical singularity if $\frac{t_{E}\left|f_{0}^{\prime}\right|}{2}$ has periodicity $2 \pi$ giving the Hawking temperature $T=\frac{1}{\beta}=\frac{\left|f_{0}^{\prime}\right|}{4 \pi}$. This regulates the IR singularity. The Lorentzian geometry as usual has an eternal extension with two asymptotic regions connected by an Einstein-Rosen bridge (wormhole), and a spacelike curvature singularity as $r \rightarrow \infty$ cloaked by the horizon as usual, with $\mathcal{R} \sim r^{1 / n-1}$.

To desingularize (11), we could also add charge and tune to extremality but this requires adding a gauge field, whereas the regulator above is contained within the theory (1).

\section{1. $\mathrm{AdS}_{D}$ gravity reduction}

The above general discussions are readily seen to hold in the theory (7), with

\footnotetext{
${ }^{1}$ We have $\Gamma_{t r}^{t}=\frac{f^{\prime}}{2}+\frac{H^{\prime}}{2 H}, \Gamma_{r r}^{r}=\frac{f^{\prime}}{2}-\frac{H^{\prime}}{2 H}, \quad \Gamma_{t t}^{r}=\frac{H}{2}\left(H f^{\prime}+H^{\prime}\right)$, $\mathcal{R}=-e^{-f}\left(H f^{\prime \prime}+H^{\prime} f^{\prime}+H^{\prime \prime}\right)$.
}

$$
n=\frac{1}{d_{i}}, \quad A=2 \Lambda=-d_{i}\left(d_{i}+1\right),
$$

which arises from the reduction of $\mathrm{AdS}_{D}$ gravity. Then the solution (11) becomes

$\phi=\frac{1}{r^{d_{i}}}, \quad d s^{2}=\frac{1}{r^{d_{i}+1}}\left(-d t^{2}+d r^{2}\right)=\frac{1}{r^{d_{i}-1}} d s_{\mathrm{AdS}_{2}}^{2}$.

where $b=-d_{i}-1, m=-d_{i}$. From (6), we see this to be the reduction of $\operatorname{AdS}_{D}$ Poincaré, i.e.,

$$
d s_{D}^{2}=\frac{1}{r^{2}}\left(-d t^{2}+d r^{2}\right)+\frac{1}{r^{2}} d x_{i}^{2},
$$

suppressing the AdS scale. The compactification from higher dimensions gives the conformal factor which leads to the IR curvature singularity in $\mathcal{R}$ (13) at the horizon $\rho=0$ (i.e., $r \rightarrow \infty$ ). Now the black hole regulator (15), (17), and in this case (20), gives the two-dimensional space

$$
\begin{aligned}
\phi & =\frac{1}{r^{d_{i}}} ; \quad d s^{2}=\frac{1}{r^{d_{i}+1}}\left(-H(r) d t^{2}+\frac{d r^{2}}{H(r)}\right), \\
H(r) & =1-\frac{r^{d_{i}+1}}{r_{0}^{d_{i}+1}} ; \quad T=\frac{d_{i}+1}{4 \pi r_{0}} .
\end{aligned}
$$

This is essentially the dimensional reduction of the $\operatorname{AdS}_{D}$ black brane.

This sort of IR horizon singularity is generic for such compactifications: for instance the reduction of global $\mathrm{AdS}_{D}$ (see Appendix A) gives

$$
\begin{aligned}
d s^{2} & =-\left(1+r^{2}\right) d t^{2}+\frac{d r^{2}}{1+r^{2}}+r^{2} d \Omega_{i}^{2} \rightarrow \phi=r^{1 / d_{i}}, \\
e^{f} & =r^{d_{i}-1}\left(-\left(1+r^{2}\right) d t^{2}+\frac{d r^{2}}{1+r^{2}}\right),
\end{aligned}
$$

with a curvature singularity in the deep interior $r \rightarrow 0$ (here $r \rightarrow \infty$ is the boundary).

Aspects of the torus reduction in (20) above were also discussed in [37] in light of generalized conformal structure; more general reductions can also be carried out where each of the torus directions is associated with a distinct warp factor (see also [41]).

\section{THE ON-SHELL ACTION AND THE HOLOGRAPHIC STRESS TENSOR}

As mentioned earlier, the on-shell action (4) for (1) has a UV divergence for $n \neq 1$. For (7), this in fact just descends from the known $\operatorname{AdS}_{D}$ on-shell action. We will now discuss the holographic energy-momentum tensor focusing on (7) which allows direct comparison with the higher 
dimensional description; many of the expressions are valid for (1) as well, with $d_{i} \rightarrow \frac{1}{n}$ as will be obvious from context. Our discussion is of course motivated by the well-known formulations of holographic renormalization [45-48] in higher dimensional theories (see also [49] in certain two-dimensional theories and [39] in nonconformal branes).

For an $A d S$-like metric, with timelike boundary $\partial \mathcal{M}$ at $r=0$ and outward pointing normal $n_{r}<0$ with $\mu$, $\nu \in \partial \mathcal{M}$, the extrinsic curvature at $\partial \mathcal{M}$ is given by

$$
\begin{aligned}
& d s_{D}^{2}=N^{2} d r^{2}+\gamma_{\mu \nu} d x^{\mu} d x^{\nu}, \quad n=-N d r, \quad K_{\mu \nu}=-\frac{1}{2}\left(\nabla_{\mu} n_{\nu}+\nabla_{\nu} n_{\mu}\right)=\Gamma_{\mu \nu}^{r} n_{r} ; \\
& d s^{2}=e^{f}\left(-d t^{2}+d r^{2}\right) \quad \Rightarrow \quad N^{2}=-\gamma_{t t}=e^{f} ; \quad K_{t t}=\frac{1}{2 N} \partial_{r} \gamma_{t t}, \quad K=\gamma^{t t} K_{t t}=\frac{f^{\prime}}{2} e^{-f / 2} .
\end{aligned}
$$

The renormalized action, comprising the bulk and the Gibbons-Hawking boundary terms, is

$$
S_{\mathrm{ren}}=\frac{1}{16 \pi G_{2}}\left[\int d^{2} x \sqrt{-g}(\phi \mathcal{R}-U(\phi))-2 \int d t \sqrt{-\gamma} \phi K-2 \int d t \sqrt{-\gamma} \phi^{\frac{d_{i}+1}{2 d_{i}}} d_{i}\right]
$$

as well as a holographic counterterm $S_{c t}$ (see also [49]), which cancels the divergences as

$S_{\text {ren }}^{\text {div }} \propto \frac{1}{16 \pi G_{2}}\left[\frac{\left(d_{i}-1\right)}{\epsilon^{d_{i}+1}}+\frac{\left(d_{i}+1\right)}{\epsilon^{d_{i}+1}}-\frac{2 d_{i}}{\epsilon^{d_{i}+1}}\right] \rightarrow 0$

thus regulating the gravitational action. Using the boundary metric from the ansatz (6), the counterterm can be seen to be just the reduction of the familiar $\operatorname{AdS}_{D}$ counterterm $\int \sqrt{h^{\left(d_{i}+1\right)}} \rightarrow \int d t \sqrt{\gamma^{(2)}} g_{i i}^{d_{i} / 2}=\int d t \sqrt{e^{f} \phi^{-\left(d_{i}-1\right) / d_{i}}} \phi$, which gives the above. The variation of the regulated action (25) with the metric can be written as [50,51]

$$
\begin{aligned}
\delta S_{\text {ren }}= & \frac{1}{16 \pi G_{2}} \int d^{2} x \sqrt{-g}\left(g_{\mu \nu} \nabla^{2} \phi-\nabla_{\mu} \nabla_{\nu} \phi+\frac{g_{\mu \nu}}{2} U\right) \delta g^{\mu \nu} \\
& -\frac{1}{16 \pi G_{2}} \int d t \sqrt{-\gamma} \gamma_{\mu \nu} n^{\rho} \nabla_{\rho} \phi \delta \gamma^{\mu \nu}+\frac{\delta S_{c t}}{\delta \gamma^{\mu \nu}} \delta \gamma^{\mu \nu} .
\end{aligned}
$$

The bulk variation term (obtained after a cancellation of second derivative terms between $\mathcal{R}$ and $K$ ) leads to the bulk equations of motion which vanish on-shell. The boundary terms above satisfy $n_{\rho} \delta \gamma^{\rho \sigma}=0$. This is consistent with [1]. Noting $n_{r}=-N$ from (24), the variation of the boundary metric then gives the holographic energy-momentum tensor as (after rescaling by the $\epsilon$-factors in $\sqrt{-\gamma} \gamma^{t t}$ to match the boundary value $\sqrt{-\hat{\gamma}} \hat{\gamma}^{t t}$ )

$T_{t t}^{\mathrm{ren}}=-\frac{2}{\sqrt{-\hat{\gamma}}} \frac{\delta S_{\mathrm{ren}}}{\delta \hat{\gamma}^{t t}}=\frac{\epsilon^{\frac{d_{i}+1}{2}}}{8 \pi G_{2}}\left(-\sqrt{g^{r r}} \partial_{r} \phi-d_{i} \phi^{\frac{d_{i}+1}{2 d_{i}}}\right) \gamma_{t t}$.

This is regarded as an expansion in $\epsilon$ where the divergent term is canceled by the counterterms leaving behind possible finite pieces with higher order pieces vanishing when the cutoff is removed as $\epsilon \rightarrow 0$. For the $\operatorname{AdS}_{D}$ reduction (20), there are no subleading terms and the counterterms have been engineered to cancel the divergences, so $T_{t t}^{\text {ren }}$ vanishes,

$$
T_{t t}^{\mathrm{ren}}=\frac{\epsilon^{\frac{d_{i}+1}{2}}}{8 \pi G_{2}}\left(-\epsilon^{\frac{d_{i}+1}{2}} \frac{\left(-d_{i}\right)}{\epsilon^{d_{i}+1}}-\frac{d_{i}}{\epsilon^{\frac{d_{i}+1}{2}}}\right) \frac{-1}{\epsilon^{d_{i}+1}}=0 .
$$

The vanishing stress tensor indicates that the background metric and dilaton define the "vacuum" of the theory; the stress tensor (28) essentially encodes excitations about this vacuum. For the black hole in this theory (22), (15), and (17), which is the reduction of the $\operatorname{AdS}_{D}$ black brane, the subleading blackening term in $g^{r r}$ gives a nonzero energymomentum

$$
\begin{aligned}
T_{t t}^{\mathrm{ren}} & =\lim _{\epsilon \rightarrow 0} \frac{\epsilon^{\frac{d_{i}+1}{2}}}{8 \pi G_{2}}\left(-\frac{d_{i}+1}{2} \frac{\left(-d_{i}\right)}{\epsilon^{d_{i}+1}} \sqrt{1-\frac{\epsilon^{d_{i}+1}}{r_{0}^{d_{1}+1}}}-\frac{d_{i}}{\epsilon^{\frac{d_{i}+1}{2}}}\right) \frac{-1}{\epsilon^{d_{i}+1}} \\
& =\frac{d_{i}}{16 \pi G_{2} r_{0}^{d_{i}+1}} .
\end{aligned}
$$

We will now discuss the holographic energy-momentum tensor in the higher dimensional theory [45-48], written in the variables of the two-dimensional theory (6) obtained after reduction. So consider

$$
\begin{aligned}
d s_{D}^{2} & =G_{r r} d r^{2}+g_{t t}^{(2)} d t^{2}+\phi^{\frac{2}{\tau_{i}}} d x_{i}^{2} \equiv G_{r r} d r^{2}+h_{\mu \nu} d x^{\mu} d x^{\nu}, \\
K_{\mu \nu} & =\Gamma_{\mu \nu}^{r} n_{r}=\frac{1}{2} \sqrt{G^{r r}} \partial_{r} h_{\mu \nu}, \\
K & =\frac{1}{2} \sqrt{G^{r r}}\left(h^{t t} \partial_{r} h_{t t}+2 \frac{\partial_{r} \phi}{\phi}\right),
\end{aligned}
$$

simplifying the $i i$ part in $K$. Then the boundary stress tensor with the usual counterterm $\sim \int d_{i} \sqrt{-h}$ (rescaled by the $\epsilon$-factors in $\sqrt{-h} h^{\mu \nu}$ to match the boundary value $\left.\sqrt{-\hat{h}} \hat{h}^{\mu \nu}\right)$ is 
$T_{\mu \nu}^{(D)}=-\frac{2}{\sqrt{-\hat{h}}} \frac{\delta S_{D}}{\delta \hat{h}^{\mu \nu}}=\frac{\epsilon^{1-d_{i}}}{8 \pi G_{D}}\left(K_{\mu \nu}-K h_{\mu \nu}-d_{i} h_{\mu \nu}\right) \rightarrow$

$T_{t t}^{(D)}=\frac{\epsilon^{1-d_{i}}}{8 \pi G_{D}}\left(-\sqrt{G^{r r}} \frac{\partial_{r} \phi}{\phi}-d_{i}\right) h_{t t}$.

Using (31) and going to the variables in the Weyltransformed frame (6) gives

$T_{t t}^{(D)}=\frac{\epsilon^{1-d_{i}}}{8 \pi G_{D}}\left(-\sqrt{g^{r r}} \partial_{r} \phi-d_{i} \phi^{\frac{d_{i}+1}{2 d_{i}}}\right) \phi^{-\frac{d_{i}+1}{2 d_{i}}} \gamma_{t t} \phi^{-\frac{d_{i}-1}{d_{i}}}$,

noting $\sqrt{G^{r r}}=\sqrt{g^{r r} \phi^{\frac{d_{i}-1}{d_{i}}}}$ and $\left.\phi\right|_{B}=\frac{1}{\epsilon^{d_{i}}}$-this matches (28). Note that reduction of the higher dimensional theory directly gives the holographic stress tensor in the two-dimensional theory; using the two-dimensional variables (6), we have

$$
\begin{aligned}
\delta S^{\text {grav }} & \sim-\frac{1}{16 \pi G_{D}} \int d^{d_{i}+1} x \sqrt{-h}\left(K_{\mu \nu}-K h_{\mu \nu}\right) \delta h^{\mu \nu} \\
& =-\frac{V_{d_{i}}}{16 \pi G_{D}} \int d t \sqrt{-\gamma^{(2)}} \phi\left(K_{t t}-K h_{t t}^{(2)}\right) \delta h_{(2)}^{t t}+\ldots \\
& =\frac{1}{16 \pi G_{2}} \int d t \sqrt{-\gamma} \sqrt{g^{r r}} \partial_{r} \phi \gamma_{t t} \delta \gamma^{t t}+\ldots
\end{aligned}
$$

noting that the $t t$ terms in $K$ cancel with $K_{t t}$ and the Weyl factors cancel between $\sqrt{G^{r r}}$ and $\sqrt{-\gamma^{(2)}}$, as well as between $h_{t t}^{(2)}$ and $\delta h_{(2)}^{t t}$. For the $\mathrm{AdS}_{D}$ black brane, (32) gives

$$
\begin{aligned}
d s^{2} & =\phi^{-\frac{d_{i}-1}{d_{i}}} e^{f}\left(-H d t^{2}+\frac{d r^{2}}{H}\right)+\phi^{\frac{2}{d_{i}}} d x_{i}^{2} \rightarrow \\
T_{t t}^{(D)} & =\frac{d_{i}}{16 \pi G_{D} r_{0}^{d_{i}+1}},
\end{aligned}
$$

matching the two-dimensional density (30) upon including the compactification volume $\frac{V_{d_{i}}}{G_{D}} \rightarrow \frac{1}{G_{2}}$.

It is useful to note that the two-dimensional energymomentum tensor calculation is in the Weyl frame where the dilaton kinetic term has been absorbed away; this matches with the higher dimensional calculation above. In a different two-dimensional Weyl frame with the dilaton kinetic term present, further holographic counterterms need to be added to cancel divergences, which then will lead to the same conclusions.

\section{A. Low-lying ("soft") modes}

It is interesting to ask if there is any analog of the $n \mathrm{AdS}_{2}$ Schwarzian action for low-lying fluctuations ("soft modes") for the Euclidean theory (7), i.e., conformally $\mathrm{AdS}_{2}$. To study this, we mimic [3] and imagine small boundary wiggles with boundary time $u$, tangent $t^{\mu}(u)$, and normal $n^{\mu}(u)$; this gives the general expression for any conformally $\mathrm{AdS}_{2}$ metric $^{2}$

$$
\begin{aligned}
K & =-\frac{t^{\mu} t^{\nu} \nabla_{\nu} n_{\nu}}{t^{\mu} t_{\mu}} \\
& =-\frac{e^{-f / 2}}{\left(\tau^{\prime 2}+r^{\prime 2}\right)^{3 / 2}}\left(r^{\prime} \tau^{\prime \prime}-\tau^{\prime} r^{\prime \prime}+\frac{f^{\prime}}{2} \tau^{\prime}\left(\tau^{\prime 2}+r^{\prime 2}\right)\right) .
\end{aligned}
$$

The natural boundary conditions on the (Euclidean) conformally $\mathrm{AdS}_{2}$ metric (20) are

$$
\frac{\tau^{\prime 2}+r^{\prime 2}}{r^{d_{i}+1}}=\frac{1}{\epsilon^{d_{i}+1}} \quad \rightarrow \quad r \sim \epsilon\left(\tau^{\prime}\right)^{\frac{2}{d_{i}+1}}
$$

to leading order. This gives ${ }^{3}$

$$
\begin{aligned}
K= & \epsilon^{\frac{d_{i}-1}{2}}\left(\tau^{\prime}\right)^{\frac{d_{i}-1}{d_{i}+1}}\left[\frac{d_{i}+1}{2}+\epsilon^{2}\left(\frac{2}{d_{i}+1}\right)\left(\tau^{\prime}\right)^{\frac{-4 d_{i}}{d_{i}+1}}\left(\tau^{\prime} \tau^{\prime \prime \prime}-\frac{5 d_{i}+1}{2\left(d_{i}+1\right)}\left(\tau^{\prime \prime}\right)^{2}\right)\right. \\
& \left.+\epsilon^{4}\left(\frac{12}{\left(d_{i}+1\right)^{3}}\right)\left(\tau^{\prime}\right)^{-\frac{8 d_{i}}{d_{i}+1}}\left(\tau^{\prime \prime}\right)^{2}\left(\tau^{\prime} \tau^{\prime \prime \prime}-\frac{9 d_{i}+1}{4\left(d_{i}+1\right)}\left(\tau^{\prime \prime}\right)^{2}\right)+\ldots\right] .
\end{aligned}
$$

Then using $\sqrt{\gamma}=e^{f / 2}, \phi=1 / r^{d_{i}}$ and (37), the Gibbons-Hawking term becomes

$$
S_{\mathrm{GH}}=-\frac{1}{8 \pi G_{2}} \int d \tau \sqrt{\gamma} \phi K=-\frac{1}{8 \pi G_{2}} \int \frac{d \tau}{r^{\left(3 d_{i}+1\right) / 2}} K=-\frac{1}{8 \pi G_{2}} \int \frac{d \tau}{\epsilon^{d_{i}+1}\left(\tau^{\prime}\right)^{2}}[\ldots]
$$

where $[\ldots]$ here is the term in square brackets in (38). For $d_{i}=1$ we see that the second term in (38) is the Schwarzian known in $n \mathrm{AdS}_{2}$. More generally we see that the second term $\left(O\left(\epsilon^{2}\right)\right)$ in (38) gives a $\frac{1}{\epsilon^{d_{i}-1}}$ divergence after sticking it into (39). Using $\nabla^{2} \phi=\frac{1}{\sqrt{\gamma}} \partial_{\tau}\left(\sqrt{\gamma} \gamma^{\tau \tau} \partial_{\tau} \phi\right)=$ $e^{-f / 2} \partial_{\tau}\left(e^{-f / 2} \partial_{\tau} \phi\right)$ as well as $\partial_{\tau}=\frac{1}{\tau^{\prime}} \partial_{u}$ and (37), we note that this can be canceled by a counterterm of the form

\footnotetext{
${ }^{2}$ For the Euclidean space $d s^{2}=e^{f}\left(d \tau^{2}+d r^{2}\right)$, we have $\Gamma_{\tau r}^{\tau}=\Gamma_{r r}^{r}=-\Gamma_{\tau \tau}^{r}=\frac{f^{\prime}}{2}=-\frac{\left(d_{i}+1\right) / 2}{r}, \quad$ and $\quad t^{\mu}=\left(\tau^{\prime}, r^{\prime}\right)$, $n^{\mu}=\frac{e^{-f / 2}}{\sqrt{\tau^{2}+r^{2}}}\left(-r^{\prime}, \tau^{\prime}\right)$, with $g_{\mu \nu} n^{\mu} n^{\nu}=1$ and $\partial_{u}=\tau^{\prime} \partial_{\tau}+r^{\prime} \partial_{r}$.

${ }^{3}$ I thank Kaberi Goswami and Hitesh Saini for correcting an error here.
} 


$$
\begin{aligned}
& \int d \tau \sqrt{\gamma} \phi^{-\frac{d_{i}+1}{2 d_{i}}}\left(-(\nabla \phi)^{2}-\phi \nabla^{2} \phi\right) \\
& \propto \int \frac{d \tau}{\epsilon^{d_{i}-1}}\left(\tau^{\prime}\right)^{\frac{-4 d_{i}}{d_{i}+1}}\left(\frac{\tau^{\prime \prime \prime}}{\tau^{\prime}}-\frac{5 d_{i}+1}{2\left(d_{i}+1\right)} \frac{\left(\tau^{\prime \prime}\right)^{2}}{\left(\tau^{\prime}\right)^{2}}\right)
\end{aligned}
$$

in the spirit of holographic renormalization. There is structural similarity with $\int \mathcal{R}$ in higher dimensions and its reduction (A3). For general $d_{i}$, there are further subleading divergences. Focusing on $d_{i}=3$ which is the $\mathrm{AdS}_{5}$ reduction, we see that the $O\left(\epsilon^{4}\right)$ term in the expansion (38) cancels the overall $\frac{1}{\epsilon^{4}}$ divergence giving a finite term at this order; this is the term in the second line in (38). Note that this $O\left(\epsilon^{4}\right)$ subleading term is at the same order as the term in (30) which gives the black hole excitation; in some ways this is in sync with the expectation of soft modes arising from the reduction of low lying hydrodynamic modes in the Super Yang-Mills conformal field theory (CFT) dual to the $\mathrm{AdS}_{5}$ black brane upstairs. It would be interesting to understand this systematically.

\section{AN EXTRA SCALAR $\Psi$}

Now we add an extra scalar field $\Psi$ to (1); one way to obtain this consistently is by reduction of the higher dimensional action $S=\frac{1}{16 \pi G_{D}} \int d^{D} x \sqrt{-g^{(D)}}$ $\left(\mathcal{R}-\frac{1}{2}(\partial \Psi)^{2}-V\right)$, which arises e.g., for nonconformal branes, $\Psi$ encoding the running gauge coupling. As before, the potential $V(g, \Psi)$ also contains metric data. The reduction (6) gives [26-28]

$S=\frac{1}{16 \pi G_{2}} \int d^{2} x \sqrt{-g}\left(\phi \mathcal{R}-U(\phi, \Psi)-\frac{1}{2} \phi(\partial \Psi)^{2}\right)$,

with $U=V \phi^{1 / d_{i}}$. The Weyl factors cancel in $(\partial \Psi)^{2}=$ $g^{\mu \nu} \partial_{\mu} \Psi \partial_{\nu} \Psi$ with $\sqrt{-g}$. The dilaton potential $U(\phi, \Psi)$ now possibly couples the dilaton $\phi$ to $\Psi$. The equations of motion are

$$
\begin{aligned}
& g_{\mu \nu} \nabla^{2} \phi-\nabla_{\mu} \nabla_{\nu} \phi+\frac{g_{\mu \nu}}{2}\left(\frac{\phi}{2}(\partial \Psi)^{2}+U\right)-\frac{\phi}{2} \partial_{\mu} \Psi \partial_{\nu} \Psi=0, \\
& \mathcal{R}-\frac{\partial U}{\partial \phi}-\frac{1}{2}(\partial \Psi)^{2}=0, \quad \frac{1}{\sqrt{-g}} \partial_{\mu}\left(\sqrt{-g} \phi \partial^{\mu} \Psi\right)-\frac{\partial U}{\partial \Psi}=0,
\end{aligned}
$$

giving

$$
\begin{aligned}
(t r) \quad \partial_{t} \partial_{r} \phi-\frac{1}{2} f^{\prime} \partial_{t} \phi-\frac{1}{2} \dot{f} \partial_{r} \phi+\frac{\phi}{2} \dot{\Psi} \Psi^{\prime} & =0, \\
(r r+t t) \quad-\partial_{t}^{2} \phi-\partial_{r}^{2} \phi+\dot{f} \partial_{t} \phi+f^{\prime} \partial_{r} \phi-\frac{\phi}{2}(\dot{\Psi})^{2}-\frac{\phi}{2}\left(\Psi^{\prime}\right)^{2} & =0, \\
(r r-t t)-\partial_{t}^{2} \phi+\partial_{r}^{2} \phi+e^{f} U & =0, \\
(\phi) \quad\left(\ddot{f}-f^{\prime \prime}\right)-\frac{1}{2}\left(-(\dot{\Psi})^{2}+\left(\Psi^{\prime}\right)^{2}\right)-e^{f} \frac{\partial U}{\partial \phi} & =0, \\
(\Psi)-\partial_{t}\left(\phi \partial_{t} \Psi\right)+\partial_{r}\left(\phi \partial_{r} \Psi\right)-e^{f} \frac{\partial U}{\partial \Psi} & =0,
\end{aligned}
$$

in conformal gauge $g_{\mu \nu}=e^{f} \eta_{\mu \nu}$. There are nontrivial dynamics in the theory (41) driven by the extra scalar $\Psi$ [28]; in particular these equations now admit nontrivial cosmological singularities which can be thought of as the reduction of higher dimensional Big Crunch singularities [52-54]. They were analyzed in [28] by using power-law scaling ansätze similar to (9), but with $\phi=t^{k} r^{m}, e^{f}=t^{a} r^{b}$, $e^{\Psi}=t^{\alpha} r^{\beta}$, in the time-dependent case. It was shown there that the near singularity behavior is universal, giving $k=1$, $a=\frac{\alpha^{2}}{2}$, the dilaton potential becoming irrelevant.
For now we will review just time-independent backgrounds; consider the potential

$$
U(\phi, \Psi)=A \phi^{n} e^{B \Psi} \quad A<0, \quad B \geq 0,
$$

in the two-dimensional theory (41). For $B=0$ and $A=2 \Lambda$, this is the same as $U$ in (9) in the theory (1). For $B \neq 0$, the form of the potential arises in e.g., nonconformal branes and more general nonrelativistic theories. With a power-law scaling ansatz, the equations (43) then give

$$
\begin{gathered}
\phi=r^{m}, \quad e^{f}=r^{b}, \quad e^{\Psi}=r^{\beta} \Rightarrow-m(m-1)+b m-\frac{\beta^{2}}{2}=0, \quad m(m-1)+A=0, \\
b+m(n-1)+B \beta+2=0, \quad b-\frac{\beta^{2}}{2}-A n=0, \quad \beta(m-1)-A B=0 .
\end{gathered}
$$


For $B=0$, these are the same as (9); we mention that there are nontrivial time-dependent solutions with $B=0$ [28]. For $B \neq 0$, we obtain

$$
\begin{aligned}
& -m(m-1)+b m-\frac{\beta^{2}}{2}=0, \quad \beta+B m=0, \quad b-\frac{\beta^{2}}{2}+m(m-1) n=0, \\
& \rightarrow b=m(n+1), \quad \beta=\sqrt{2 m(m n+1)}, \quad B=\sqrt{\frac{2(m n+1)}{m}} \Rightarrow m=\frac{2}{B^{2}-2 n} .
\end{aligned}
$$

We know that $\phi$ grows as $r$ decreases so $m<0$; this is consistent with $m(m-1)=-A>0$ and gives nontrivial consistency conditions for the existence of these backgrounds

$B^{2}<2 n, \quad \frac{2}{B^{2}-2 n}\left(\frac{2}{B^{2}-2 n}-1\right)=-A>0$.

(The first condition is also consistent with null energy conditions.) Our discussion here is bottom-up. Of course these conditions are all satisfied in the reduction of known higher dimensional theories as noted in [28]; e.g., we have $n=\frac{1}{d_{i}}$ and $B=\sqrt{\frac{2(-\theta)}{d_{i}\left(d_{i}-\theta\right)}}$ with $\theta \leq 0$, and $m=-\left(d_{i}-\theta\right)$, $b=-\left(d_{i}-\theta\right)\left(1+\frac{1}{d_{i}}\right)$; also $A=\left(d_{i}-\theta\right)\left(d_{i}+1-\theta\right)$. The higher dimensional space is then (14). As stated there, some of the known reductions include those of nonconformal $D p$-branes: the two-dimensional geometries regulated with a blackening factor are then the reductions of black nonconformal $D p$-branes [24].

Now we make a few comments on the energymomentum tensor in (41). From the two-dimensional equations of motion (42), we have $\mathcal{R}=\partial_{\phi} U+\frac{1}{2}(\partial \Psi)^{2}$. Thus the $(\partial \Psi)^{2}$ terms cancel on-shell if the potential $U(\phi)$ does not contain $\Psi$, then the scalar has entirely disappeared

from the on-shell action, which is in fact of the same form as (25) (in particular the same counterterm there suffices). We will not discuss more complicated dilaton potentials $U(\phi, \Psi)$ here.

Now we consider the two-dimensional cosmology obtained from the $\mathrm{AdS}_{D}$ Kasner reduction in [28]

$$
\begin{aligned}
d s^{2} & =\frac{1}{r^{2}}\left(-d t^{2}+d r^{2}\right)+\frac{t^{2 p_{i}}}{r^{2}} d x_{i}^{2}, \quad e^{\Psi}=t^{\alpha} \\
\rightarrow \quad \phi & =\frac{t}{r^{d_{i}}}, \quad d s^{2}=\frac{t^{\left(d_{i}-1\right) / d_{i}}}{r^{d_{i}+1}}\left(-d t^{2}+d r^{2}\right), \\
e^{\Psi} & =t^{\sqrt{2\left(d_{i}-1\right) / d_{i}}} .
\end{aligned}
$$

Noting that the extra scalar required to drive these cosmologies has disappeared in the on-shell action, we will see from (28) that the energy-momentum tensor $T_{t t}^{r e n}$ vanishes. The scaling ansatz $\phi=t^{k} r^{m}, e^{f}=t^{a} r^{b}, e^{\Psi}=t^{\alpha} r^{\beta}$, present in these cosmological backgrounds shows that the $t$-dependence in (48) appears solely in terms of multiplicative factors over the $\operatorname{AdS}_{D}$ background profile (20) itself. Thus the holographic energy-momentum tensor (28) is

$$
\begin{aligned}
d s^{2} & =e^{f}\left(-d t^{2}+d r^{2}\right), \quad e^{f}=\frac{t^{a}}{r^{d_{i}+1}}, \quad \phi=\frac{t^{k}}{r^{d_{i}}} ; \quad a=\frac{d_{i}-1}{d_{i}}, \quad k=1, \\
T_{t t}^{\mathrm{ren}} & =\frac{\epsilon^{\frac{d_{i}+1}{2}}}{8 \pi G_{2}}\left(-\frac{d_{i}+1}{2} \frac{\left(-d_{i}\right)}{\epsilon^{d_{i}+1}}\left(t^{-\frac{a}{2}+k}\right)-\frac{d_{i}}{\epsilon^{\frac{d_{i}+1}{2}}}\left(t^{\frac{k\left(d_{i}+1\right)}{2 d_{i}}}\right)\right) \frac{-1}{\epsilon^{d_{i}+1}}=0 .
\end{aligned}
$$

The $t$-dependencies in both factors are in fact identical since the algebraic conditions on the exponents following from the equations of motion [28] give $a=k\left(\frac{d_{i}-1}{d_{i}}\right)$ which is seen to be satisfied above. Thus this of the same form as (29) apart from identical $t$-factors in each term and leads to $T_{t t}^{\mathrm{ren}}=0$. This is also corroborated by (32) in the higher dimensional $\mathrm{AdS}_{D}$ Kasner spacetime [52-54]; we see that the t-dependence disappears in $\phi$-terms and $g^{r r}$ has none. Further the other counterterms discussed in [53] also cancel in this holographic screen so finally $T_{t t}^{(D)}=0$. Note that each $t$-factor has a positive exponent so in the vicinity of the singularity at $t=0$ each term independently becomes vanishingly small. Overall this suggests a kind of finetuning to the energy-momentum tensor in these holographic screens; although the bulk fields (in particular the metric) have nonvanishing non-normalizable deformations turned on, the response vanishes suggesting that the state is nongeneric. However this is screen dependent; a Penrose-Brown-Henneaux transformation recasting the metric schematically as $d s^{2}=\frac{t^{a}}{r^{d_{i}+1}}\left(-d t^{2}+d r^{2}\right) \equiv$ $d R^{2}\left(\frac{1}{R^{d_{i}+1}}+\ldots\right)+\gamma_{T T}(T, R) d T^{2}$ appears to lead to a nonvanishing energy-momentum tensor. A more detailed study 
of this would presumably yield analogs of the corresponding findings in [53].

\section{A. Some comments on scalar probes}

Now we will discuss aspects of massless scalars regarded as probes; we will focus on the theory (7) obtained from $\mathrm{AdS}_{D}$ reduction, so as to facilitate direct comparison of the two-dimensional and higher dimensional discussions. To generalize to (1) with a general monomial potential, it is adequate to set $d_{i} \rightarrow \frac{1}{n}$ in most expressions.

A massless scalar probe $\psi$ descending from higher dimensions has action and equation of motion

$-\int d^{2} x \sqrt{g} \phi_{B}(\partial \psi)^{2} \rightarrow \frac{1}{\sqrt{g}} \partial_{\mu}\left(\phi_{B} \sqrt{g} g^{\mu \nu} \partial_{\nu} \psi\right)=0$,

focusing on the Euclidean theory; here the background dilaton profile $\phi_{B}$ is nonconstant. For a time-independent probe in the background (20), we obtain

$$
\begin{aligned}
\partial_{r}\left(\phi_{b} \partial_{r} \psi\right) & =0=\partial_{r}\left(r^{-d_{i}} \partial_{r} \psi\right)=0 \quad \rightarrow \quad \partial_{r} \psi=\frac{C_{1}}{\phi_{B}} \\
\rightarrow \quad \psi & =C_{0}+C_{1} r^{d_{i}+1} .
\end{aligned}
$$

So for $d_{i}>1$, the scalar probe dies rapidly towards the boundary. The scalar probe perturbation modifies the background potential which can be read off from the effective action as

$$
\begin{gathered}
\int\left(\phi \mathcal{R}-U(\phi)-\frac{1}{2} \phi g^{r r}\left(\partial_{r} \psi\right)^{2}\right) \\
\Rightarrow \quad U\left(\phi_{B}\right) \rightarrow U\left(\phi_{B}\right)-\frac{C_{1}^{2} e^{-f_{B}}}{2 \phi_{B}} .
\end{gathered}
$$

This can now be used to understand backreaction; defining $\phi=\phi_{B}+\varphi$, gives from (43)

$$
\partial_{r}^{2} \varphi=-e^{f_{B}} \delta U=\frac{C_{1}^{2}}{2 \phi_{B}} \quad \rightarrow \quad \varphi \sim C_{1}^{2} r^{d_{i}+2},
$$

which as $r \rightarrow 0$ is a small correction to the background dilaton $\phi_{B} \sim \frac{1}{r^{d_{i}}}$ so that the asymptotic background (20) does not suffer large backreaction. Equivalently the background can support small excitations.

Now consider operators dual to scalar probes in the Euclidean space (20); (50) gives

$$
\begin{aligned}
0 & =\partial_{\mu}\left(\phi \sqrt{g} g^{\mu \nu} \partial_{\nu} \psi\right)=\partial_{\tau}\left(\phi \partial_{\tau} \psi\right)+\partial_{r}\left(\phi \partial_{r} \psi\right) \\
& \rightarrow \quad \partial_{r}\left(\frac{1}{r^{d_{i}}} \partial_{r} \psi_{\omega}\right)-\frac{1}{r^{d_{i}}} \omega^{2} \psi_{\omega}=0
\end{aligned}
$$

with $\psi(\tau, r)=e^{-i \omega \tau} \psi_{\omega}(r)$. This has many similarities with the higher dimensional $\operatorname{AdS}_{D}$ but it is instructive to look at the simplest nontrivial case $d_{i}=2$ in detail; then $\left(\nu=\frac{d_{i}+1}{2}\right)$

$$
\psi_{\omega}=\psi_{\omega}^{0} \frac{r^{\left(d_{i}+1\right) / 2} K_{\nu}(\omega r)}{\epsilon^{\left(d_{i}+1\right) / 2} K_{\nu}(\omega \epsilon)} \stackrel{d_{i} \rightarrow 2}{\longrightarrow} \psi_{\omega}^{0} \frac{(1+\omega r) e^{-\omega r}}{(1+\omega \epsilon) e^{-\omega \epsilon}},
$$

which is regular at large $r$ although the two-dimensional space is singular. Using the bulk equation, the on-shell action gives a boundary term as usual. Expanding in $\epsilon$ gives

$$
\begin{aligned}
-S & \left.\sim \int d \omega \frac{1}{\epsilon^{d_{i}}} \psi_{-\omega} \partial_{r} \psi_{\omega}\right|_{\epsilon} \rightarrow \int \frac{d \omega}{\epsilon^{2}} \psi_{-\omega}^{0} \psi_{\omega}^{0} \frac{-\omega^{2} \epsilon}{(1+\omega \epsilon)} \\
& =\int d \omega \psi_{-\omega}^{0} \psi_{\omega}^{0}\left(\frac{-\omega^{2}}{\epsilon}+\omega^{3}+\ldots\right) .
\end{aligned}
$$

The first term can be removed by a local counterterm $\int d \tau \sqrt{\gamma} \phi^{5 / 4}(\partial \Psi)_{B}^{2}$; this in fact arises from the higher dimensional $\int d \tau d^{d_{i}} x \sqrt{h} h^{i j} \partial_{i} \Psi \partial_{j} \Psi \rightarrow$ $\int d t \sqrt{\gamma^{(2)}} \phi \gamma^{(2) \tau \tau}\left(\partial_{\tau} \Psi\right)^{2}$ after incorporating the Weyl transformation (6). The second nonlocal term is the same momentum space two-point function as in $\mathrm{AdS}_{4}$. Fourier transforming, now in $(0+1)$ dimensions, gives in position space

$$
\left\langle O(\tau) O\left(\tau^{\prime}\right)\right\rangle \sim \int d \omega e^{i \omega \Delta \tau} \omega^{3} \sim \frac{1}{(\Delta \tau)^{4}},
$$

giving the operator dimension $\Delta=2$ for $O(\tau)$. For $d_{i}>1$, the short-time divergence here $(\Delta \tau \rightarrow 0)$ is more severe than for a dimension $\Delta=1$ operator as would be the case if the dilaton factor were absent in (50). This can be extended to $d_{i}>2$ also; the momentum space answer is identical to the corresponding calculation in higher dimensional $\operatorname{AdS}_{D}$ giving $\omega^{2 \nu}(\log \omega \epsilon)$ (including the possible logarithmic piece for $\nu \in \mathbb{Z}$ ) but the one-dimensional Fourier transform gives the position space correlator $\frac{1}{(\Delta \tau)^{d_{i}+2}}$ and thereby $1+\frac{d_{i}}{2}$ for the dimension of the operator $O(\tau)$, which is distinct from $\mathrm{AdS}_{d_{i}+2}$ where the operator dimension would have been $d_{i}+1$, from the $\left(d_{i}+1\right)$ dimensional Fourier transform (see also [37]).

It is interesting that the above two-point correlation function calculation is insensitive to the singularity in the zero temperature two-dimensional space, stemming from regularity of the bulk mode functions. It is likely that this is a generic feature, i.e., low point correlators do not see the singularity in the zero temperature two-dimensional space. It would be interesting to understand this better.

It would seem overall that we have just scratched the surface here. In light of (53) one might think backreaction effects are small; it would be interesting to study correlation functions in greater detail, incorporating possible time reparametrizations along the lines of [1]. Modes with nontrivial dilaton couplings may also be of interest in 
understanding aspects of the Sachdev-Ye-Kitaev theory (see, for example, the recent work [55]).

\section{DISCUSSION}

We have discussed aspects of generic two-dimensional dilaton gravity theories bottom-up. The two-dimensional geometry is in general conformal to $\mathrm{AdS}_{2}$ and has IR curvature singularities at zero temperature, which are regulated by a black hole. As we saw, the on-shell action is divergent: we discussed the holographic energymomentum tensor by adding appropriate counterterms. For theories obtained by dimensional reduction of the gravitational sector of higher dimensional theories, the two-dimensional description dovetails with the higher dimensional one, as we saw explicitly in the reduction of higher dimensional AdS gravity as a concrete example. The analysis of low-lying ("soft") modes reveals distinct departures from the $n \mathrm{AdS}_{2}$ Schwarzian, stemming from further divergences here (38). Overall, such two-dimensional theories appear to encode higher dimensional gravity intrinsically (there are parallels with old work e.g., [56], also reviews e.g., [29]). This is quite different from near extremal objects with $\mathrm{AdS}_{2} \times X$ throats; here the $X$-compactification leads to JT gravity which is intrinsically two-dimensional (and topological in some sense). We also discussed adding an extra scalar field which drives nontrivial dynamics, in particular the twodimensional cosmological singularities discussed in [28]: the new dynamical direction that the scalar defines may have similarities with that in backgrounds that include rotation, gauge fields etc, (see, for example, [57,58]). For scalar probes with dilaton couplings, we saw that correlation functions of dual operators (56) and (57), are insensitive to the singularity in the zero temperature two-dimensional space, a feature likely to extend to more general low point correlators.

We now recall the discussion in [11] of JT gravity as dual to a random matrix ensemble; further developments appear in [12-21]. In this light, the perturbative studies here suggest that generic two-dimensional dilaton gravity theories (1) are somewhat different from JT gravity and theories "near JT", e.g., through the behavior of IR singularities in the zero temperature two-dimensional geometry and the divergence in the on-shell action. Nonperturbatively in JT gravity, we recall that the $\phi$ path integral leads to a sum over constant curvature surfaces of various topologies (which maps to a corresponding expansion of a matrix integral). By comparison for a more general potential in the Euclidean theory, we have $\int D g D \phi \exp \left[\int \sqrt{g}(\phi \mathcal{R}-U)\right]$ which using the dilaton equation in (2) and noting (4) naively leads to $\sim \int D g \exp \left[\# \int \sqrt{g}(-\mathcal{R})^{-1 /\left(d_{i}-1\right)}\right]$ in the specific case (7), with \# a positive number. By comparison, a simple Gaussian potential $U=\lambda \phi^{2}$ gives $\sim \int D g \exp \left[\# \int \sqrt{g} \frac{\mathcal{R}^{2}}{\lambda}\right]$ after doing the gaussian $\phi$-integral, which is in two-dimensional $\mathcal{R}^{2}$ gravity. These structures appear more intricate (also, the sign of $U$ enters nontrivially); it would be interesting to understand them in greater depth, perhaps towards mapping to some effective matrix model.

It would appear that two-dimensional theories such as (1) are not "near JT" in the sense of the deformations of JT gravity in $[15,16]$; roughly $\left|U-U_{J T}\right|$ here is not sufficiently small asymptotically, unlike there. As a concrete example, the theory (7) for $d_{i}>1$ in some essential sense faithfully reflects its higher dimensional origins. Ordinarily we regard gravity in dimensions $D \geq 4$ as UV completed by a string/M theory; a particular gauge/gravity dual pair is usually pinned down by specifying the precise matter content, including information on $X$ (so the bulk string theory is then critical). From this point of view, in treating the $\mathrm{AdS}_{D}$ gravity reduction (7) as a stand alone theory, we have effectively performed some sort of average or partial trace (loosely speaking) over the UV information that encodes the identity of the specific gauge/gravity dual pair that it was compactified from (equivalently, with some partial trace over local operators from the dual field theory point of view). In this light, it would perhaps be interesting to understand minimal string theories which are twodimensional to begin with and their dual matrix models (see e.g., [59], as well as older reviews e.g., [60,61]) and the possibly distinct kinds of embeddings of such two-dimensional gravity theories obtained thereby (see e.g., $[11,12,18]$ for some discussions, as well as e.g., [62]). Since the generic theory (1) is structurally similar to (7), this suggests that the generic two-dimensional theory (1) is a UV incomplete low energy effective theory, akin to a thermodynamic ensemble in a sense (naively the dual operators are simply $T_{t t}^{(1)}$ and $T_{\phi} \equiv T_{i i}$; in the case of a higher dimensional CFT compactified spatially, these are subject to the tracelessness condition, thus giving $T_{t t}^{(1)}$ alone in the effective one-dimensional dual, with no microstate information). While this is adequate for understanding various holographic (large $N$ ) observables as we have seen, the nature of such a thermodynamic ensemble appears fundamentally different from the ensemble dual to JT. The two-dimensional theories here represent some sort of "effective holography", i.e., some approximate gauge/ gravity duality, with a subset of observables; it would be interesting to understand more systematically such incomplete holographic models, as well as their validity and usefulness. There are some parallels with the discussions in [14] of higher dimensional gravity. The case $d_{i}=1$ (7) is the $\mathrm{AdS}_{3}$ reduction, which as we have seen has many parallels with JT gravity; this appears in accord with [17]. It would be interesting to understand these issues better and gain more insight into quantifying the ensemble nature of such effective generic two-dimensional gravity theories (at least those that are adequately well defined intrinsically or via reductions from reasonable theories upstairs, and not in 
some sort of swampland) and possible effective matrix models, and the role of replica wormholes [63,64] here.

\section{ACKNOWLEDGMENTS}

It is a pleasure to thank Sumit Das, Tom Hartman, Dileep Jatkar, Igor Klebanov, Kedar Kolekar, Arnab Kundu, Alok Laddha, Henry Maxfield and Douglas Stanford for some early correspondence around Strings, helpful discussions and comments on a draft, and the organizers of the virtual Strings 2020 for a stimulating conference. This work is partially supported by a grant to CMI from the Infosys Foundation.

\section{APPENDIX: REDUCTION DETAILS}

$T^{D-2}$ reduction: The $D$-dimensional space with $D=$ $d_{i}+2$ is of the form (6); we have

$$
\begin{aligned}
& \int \frac{d^{D} x}{G_{D}} \sqrt{-g^{(D)}}\left(\mathcal{R}^{(D)}-V\right) \\
& \rightarrow \int \frac{d^{2} x}{G_{2}} \sqrt{-g^{(2)}}\left(\phi \mathcal{R}^{(2)}+\frac{D-3}{D-2} \frac{(\partial \phi)^{2}}{\phi}-V \phi\right),
\end{aligned}
$$

from reduction to the two-dimensional space $g^{(2)}$. A total derivative from the reduction cancels one from the reduction of the Gibbons-Hawking term. As in [1] and [26,27], noting

$$
\begin{aligned}
& \sqrt{-g^{(2)}}\left[\phi \mathcal{R}^{(2)}+\lambda \frac{(\partial \phi)^{2}}{\phi}\right] \\
& \stackrel{g_{a b}^{(2)}=\phi^{-\alpha} g_{a b}}{\longrightarrow} \sqrt{-g}\left[\phi \mathcal{R}+(\lambda-\alpha) \frac{(\partial \phi)^{2}}{\phi}\right],
\end{aligned}
$$

we use the Weyl transformation in (6) to absorb the dilaton kinetic term into $\mathcal{R}$, giving (7); the factor in $V$ gives $\phi^{-\frac{D-3}{D-2}+1}$.

$S^{D-2}$ reduction: Here the curvature of the sphere gives additional terms (see e.g., [29-32]); we have

$$
\begin{aligned}
R^{(D)}= & R^{(2)}+\frac{(D-3)(D-2) \phi^{\frac{D-4}{D-2}}}{\phi} \\
& +\frac{D-3}{D-2} \frac{\left(\partial_{(2)} \phi\right)^{2}}{\phi^{2}}-2 \frac{\nabla_{(2)}^{2} \phi}{\phi},
\end{aligned}
$$

from (6) with $d \sigma_{D-2}^{2} \equiv d \Omega_{D-2}^{2}$. Then reduction and the Weyl transformation (6) gives

$$
\begin{array}{r}
\int d^{2} x \sqrt{-g^{(2)}}\left(\phi \mathcal{R}^{(2)}+\frac{D-3}{D-2} \frac{(\partial \phi)^{2}}{\phi}+(D-2)(D-3) \phi^{\frac{D-4}{D-2}}-V \phi\right) \\
\stackrel{V=2 \Lambda}{\longrightarrow} \int d^{2} x \sqrt{-g}\left(\phi \mathcal{R}+d_{i}\left(d_{i}+1\right) \phi^{1 / d_{i}}+d_{i}\left(d_{i}-1\right) \phi^{-1 / d_{i}}\right) .
\end{array}
$$

For global $\mathrm{AdS}_{D}$, the reduction (6) of the background gives

$$
\begin{aligned}
d s^{2} & =-\left(1+r^{2}\right) d t^{2}+\frac{d r^{2}}{1+r^{2}}+r^{2} d \Omega_{i}^{2} \\
\rightarrow \quad \phi & =r^{d_{i}}, \quad e^{f}=r^{d_{i}-1}\left(-\left(1+r^{2}\right) d t^{2}+\frac{d r^{2}}{1+r^{2}}\right),
\end{aligned}
$$

with curvature $\mathcal{R}=-r^{-d_{i}-1}\left(\left(d_{i}+1\right) r^{2}-\left(d_{i}-1\right)\right)$. Thus $\mathcal{R} \sim \frac{1}{r^{d i-1}}$ as $r \rightarrow 0$ (interior) so the singularity structure is similar to that for the $\operatorname{AdS}_{D}$ Poincare reduction. The AdS Schwarzschild black hole under the reduction (6) gives

$$
\begin{aligned}
\phi & =r^{d_{i}} \\
e^{f} & =r^{d_{i}-1}\left(-\left(1+r^{2}-\frac{1}{r^{d_{i}-1}}\right) d t^{2}+\frac{d r^{2}}{1+r^{2}-\frac{1}{r^{d_{i}-1}}}\right) .
\end{aligned}
$$


[1] A. Almheiri and J. Polchinski, Models of $\mathrm{AdS}_{2}$ backreaction and holography, J. High Energy Phys. 11 (2015) 014.

[2] A. Kitaev, A simple model of quantum holography, in Proceedings at the KITP, Santa Barbara, 2015, http:// online.kitp.ucsb.edu/online/entangled15/kitaev/, http://online .kitp.ucsb.edu/online/entangled15/kitaev2/.

[3] J. Maldacena, D. Stanford, and Z. Yang, Conformal symmetry and its breaking in two dimensional nearly Anti-deSitter space, Prog. Theor. Exp. Phys. 2016, 12C104 (2016).

[4] K. Jensen, Chaos in $\mathrm{AdS}_{2}$ Holography, Phys. Rev. Lett. 117, 111601 (2016).

[5] J. Engelsy, T. G. Mertens, and H. Verlinde, An investigation of $\mathrm{AdS}_{2}$ backreaction and holography, J. High Energy Phys. 07 (2016) 139.

[6] G. Srosi, $\mathrm{AdS}_{2}$ holography and the SYK model, Proc. Sci., Modave2017 (2018) 001 [arXiv:1711.08482].

[7] V. Rosenhaus, An introduction to the SYK model, J. Phys. A 52, 323001 (2019).

[8] D. A. Trunin, Pedagogical introduction to SYK model and 2D dilaton gravity, Usp. Fiz. Nauk 191, 225 (2021).

[9] R. Jackiw, Lower dimensional gravity, Nucl. Phys. B252, 343 (1985).

[10] C. Teitelboim, Gravitation and Hamiltonian structure in two space-time dimensions, Phys. Lett. 126B, 41 (1983).

[11] P. Saad, S. H. Shenker, and D. Stanford, JT gravity as a matrix integral, arXiv:1903.11115.

[12] D. Stanford and E. Witten, JT gravity and the ensembles of random matrix theory, arXiv:1907.03363.

[13] D. Marolf and H. Maxfield, Transcending the ensemble: Baby universes, spacetime wormholes, and the order and disorder of black hole information, J. High Energy Phys. 08 (2020) 044.

[14] J. McNamara and C. Vafa, Baby universes, holography, and the Swampland, arXiv:2004.06738.

[15] E. Witten, Deformations of JT gravity and phase transitions, arXiv:2006.03494.

[16] E. Witten, Matrix models and deformations of JT gravity, Proc. R. Soc. A 476, 20200582 (2020).

[17] H. Maxfield and G. J. Turiaci, The path integral of 3D gravity near extremality; or, JT gravity with defects as a matrix integral, J. High Energy Phys. 01 (2021) 118.

[18] C. V. Johnson, Explorations of nonperturbative JT gravity and supergravity, Phys. Rev. D 103, 046013 (2021).

[19] Y. Kimura, JT gravity and the asymptotic Weil-Petersson volume, Phys. Lett. B 811, 135989 (2020).

[20] D. Stanford, More quantum noise from wormholes, arXiv:2008.08570.

[21] M. Alishahiha, A. Faraji Astaneh, G. Jafari, A. Naseh, and B. Taghavi, On free energy for deformed JT gravity, Phys. Rev. D 103, 046005 (2021).

[22] M. Taylor, Lifshitz holography, Classical Quantum Gravity 33, 033001 (2016).

[23] S. A. Hartnoll, A. Lucas, and S. Sachdev, Holographic quantum matter, arXiv:1612.07324.

[24] N. Itzhaki, J. M. Maldacena, J. Sonnenschein, and S. Yankielowicz, Supergravity and the large N limit of theories with sixteen supercharges, Phys. Rev. D 58, 046004 (1998).

[25] X. Dong, S. Harrison, S. Kachru, G. Torroba, and H. Wang, Aspects of holography for theories with hyperscaling violation, J. High Energy Phys. 06 (2012) 041.
[26] K. S. Kolekar and K. Narayan, $\mathrm{AdS}_{2}$ dilaton gravity from reductions of some nonrelativistic theories, Phys. Rev. D 98, 046012 (2018).

[27] K. S. Kolekar and K. Narayan, On $\mathrm{AdS}_{2}$ holography from redux, renormalization group flows and c-functions, J. High Energy Phys. 02 (2019) 039.

[28] R. Bhattacharya, K. Narayan, and P. Paul, Cosmological singularities and 2-dimensional dilaton gravity, J. High Energy Phys. 08 (2020) 062.

[29] A. Strominger, Les Houches lectures on black holes, arXiv: hep-th/9501071.

[30] S. Nojiri and S. D. Odintsov, Quantum dilatonic gravity in $(D=2)$-dimensions, $(D=4)$-dimensions and $(D=5)$ dimensions, Int. J. Mod. Phys. A 16, 1015 (2001).

[31] D. Grumiller, Quantum dilaton gravity in two-dimensions with matter, arXiv:gr-qc/0105078.

[32] D. Grumiller, W. Kummer, and D. V. Vassilevich, Dilaton gravity in two-dimensions, Phys. Rep. 369, 327 (2002).

[33] J. M. Maldacena, The large N limit of superconformal field theories and supergravity, Adv. Theor. Math. Phys. 2, 231 (1998); Int. J. Theor. Phys. 38, 1113 (1999).

[34] S. S. Gubser, I. R. Klebanov, and A. M. Polyakov, Gauge theory correlators from noncritical string theory, Phys. Lett. B 428, 105 (1998).

[35] E. Witten, Anti-de Sitter space and holography, Adv. Theor. Math. Phys. 2, 253 (1998).

[36] O. Aharony, S. S. Gubser, J. M. Maldacena, H. Ooguri, and Y. Oz, Large $\mathrm{N}$ field theories, string theory and gravity, Phys. Rep. 323, 183 (2000).

[37] M. Taylor, Generalized conformal structure, dilaton gravity and SYK, J. High Energy Phys. 01 (2018) 010.

[38] A. Jevicki, Y. Kazama, and T. Yoneya, Generalized conformal symmetry in D-brane matrix models, Phys. Rev. D 59, 066001 (1999).

[39] I. Kanitscheider, K. Skenderis, and M. Taylor, Precision holography for nonconformal branes, J. High Energy Phys. 09 (2008) 094.

[40] I. Kanitscheider and K. Skenderis, Universal hydrodynamics of nonconformal branes, J. High Energy Phys. 04 (2009) 062.

[41] B. Gouteraux, J. Smolic, M. Smolic, K. Skenderis, and M. Taylor, Holography for Einstein-Maxwell-dilaton theories from generalized dimensional reduction, J. High Energy Phys. 01 (2012) 089.

[42] M. Cavaglia, Geometrodynamical formulation of twodimensional dilaton gravity, Phys. Rev. D 59, 084011 (1999).

[43] J. Maldacena, G. J. Turiaci, and Z. Yang, Two dimensional nearly de Sitter gravity, J. High Energy Phys. 01 (2021) 139.

[44] K. Narayan, On Lifshitz scaling and hyperscaling violation in string theory, Phys. Rev. D 85, 106006 (2012).

[45] V. Balasubramanian and P. Kraus, A stress tensor for anti-de Sitter gravity, Commun. Math. Phys. 208, 413 (1999).

[46] R. C. Myers, Stress tensors and Casimir energies in the AdS/CFT correspondence, Phys. Rev. D 60, 046002 (1999).

[47] S. de Haro, S. N. Solodukhin, and K. Skenderis, Holographic reconstruction of spacetime and renormalization in the AdS/CFT correspondence, Commun. Math. Phys. 217, 595 (2001). 
[48] K. Skenderis, Lecture notes on holographic renormalization, Classical Quantum Gravity 19, 5849 (2002).

[49] M. Cvetič and I. Papadimitriou, $\mathrm{AdS}_{2}$ holographic dictionary, J. High Energy Phys. 12 (2016) 008; Erratum, J. High Energy Phys. 01 (2017) 120.

[50] Kedar Kolekar, Ph.D. thesis, CMI Chennai, 2019.

[51] D. Grumiller, R. McNees, J. Salzer, C. Valcárcel, and D. Vassilevich, Menagerie of $\mathrm{AdS}_{2}$ boundary conditions, J. High Energy Phys. 10 (2017) 203.

[52] S. R. Das, J. Michelson, K. Narayan, and S. P. Trivedi, Time dependent cosmologies and their duals, Phys. Rev. D 74, 026002 (2006).

[53] A. Awad, S. R. Das, K. Narayan, and S. P. Trivedi, Gauge theory duals of cosmological backgrounds and their energy momentum tensors, Phys. Rev. D 77, 046008 (2008).

[54] A. Awad, S. Das, S. Nampuri, K. Narayan, and S. Trivedi, Gauge theories with time dependent couplings and their cosmological duals, Phys. Rev. D 79, 046004 (2009).

[55] S. R. Das, A. Ghosh, A. Jevicki, and K. Suzuki, Near conformal perturbation theory in SYK type models, J. High Energy Phys. 12 (2020) 171.
[56] S. B. Giddings and A. Strominger, Quantum theories of dilaton gravity, Phys. Rev. D 47, 2454 (1993).

[57] A. Castro, F. Larsen, and I. Papadimitriou, 5D rotating black holes and the $\mathrm{nAdS}_{2} / \mathrm{nCFT}_{1}$ correspondence, J. High Energy Phys. 10 (2018) 042.

[58] U. Moitra, S. P. Trivedi, and V. Vishal, Extremal and nearextremal black holes and near-CFT 1 , J. High Energy Phys. 07 (2019) 055.

[59] M. R. Douglas, I. R. Klebanov, D. Kutasov, J. M. Maldacena, E. J. Martinec, and N. Seiberg, A new hat for the $c=1$ matrix model, arXiv:hep-th/0307195.

[60] I. R. Klebanov, String theory in two-dimensions, arXiv:hepth/9108019.

[61] P. H. Ginsparg and G. W. Moore, Lectures on 2-D gravity and 2-D string theory, arXiv:hep-th/9304011.

[62] P. Betzios and O. Papadoulaki, Liouville theory and Matrix models: A Wheeler DeWitt perspective, J. High Energy Phys. 09 (2020) 125.

[63] G. Penington, S. H. Shenker, D. Stanford, and Z. Yang, Replica wormholes \& the black hole interior, arXiv:1911.11977.

[64] A. Almheiri, T. Hartman, J. Maldacena, E. Shaghoulian, and A. Tajdini, Replica wormholes and the entropy of Hawking radiation, J. High Energy Phys. 05 (2020) 013. 